\title{
Determination of optimum harvest maturity and non-destructive evaluation of pod development and maturity in cacao (Theobroma cacao L.) using a multiparametric fluorescence sensor
}

\begin{abstract}
BACKGROUND: A series of fluorescence indices (anthocyanin, flavonol, chlorophyll and nitrogen balance) were deployed to detect the pigments and colourless flavonoids in cacao pods of three commercial cacao (Theobroma cacao L.) genotypes (QH1003, KKM22 and MCBC1) using a fast and non-destructive multiparametric fluorescence sensor. The aim was to determine optimum harvest periods (either 4 or 5 months after pod emergence) of commercial cacao based on fluorescence indices of cacao development and bean quality.

RESULTS: As pod developed, cacao exhibited a rise with the peak of flavonol occurring at months 4 and 5 after pod maturity was initiated while nitrogen balance showed a decreasing trend during maturity. Cacao pods contained high chlorophyll as they developed but chlorophyll content declined significantly on pods that ripened at month 5 .

CONCLUSION: Cacao pods harvested at months 4 and 5 can be considered as commercially-ready as the beans have developed good quality and comply with the Malaysian standard on cacao bean specification. Thus, cacao pods can be harvested earlier when they reach maturity at month 4 after pod emergence to avoid germinated beans and over fermentation in ripe pods harvested at month 5.
\end{abstract}

Keyword: Cacao (Theobroma cacao L.); Non-destructive; Fluorescence-based indices; Bean quality; Cacao maturity 